\title{
Percepción local del proceso de Etnoeducación en La Chorrera, Amazonas -Colombia ${ }^{1}$
}

\section{Local perception of the etnoeducation process in La Chorrera- Amazon Basin - Colombia}

\section{Por: Farekatde-Maribba Gi1² ; Henao-Mejía Luis Guillermo ${ }^{3}$}

\section{Resumen}

La etnoeducación en La Chorrera ha tenido un proceso de maduración, desde los primeros intentos oficiales a una postura moderna basada en el sincretismo cultural. Se presentan las bases de una etnoeducación propia, discutida con los ancianos, basada en los ciclos naturales de la Amazonia y proclamada como ley de vida: Pensar - palabra - obra. En la educación tradicional de la cultura del tabaco, están reguladas cuatro etapas del conocimiento y de la vida: escuchar, practicar, enseñar y cuidar.

Palabras claves: etnoeducación, educación tradicional, cultura del "tabaco, la coca y la yuca dulce"

\begin{abstract}
Ethnoeducation in La Chorrera has been ripped from the former governmental initiatives to a modern one based in intercultural syncretism. We present the bases of a self ethnoeducation, enriched from the community's elders, based in the natural cycles of the Amazon: the life's law of "word - think - opus". In the tobacco cultures traditional education there are regulations for the four steps in knowledge and life: hearing, practicing, teaching and caring.
\end{abstract}

Key words: ethnoeducation, traditional education, tobacco, coca and sweet manioc culture

Las expresiones en bastardilla están en uitoto $m+n+k a$, a menos que se diga otra cosa.

La etnoeducación planteada desde el sector oficial, principalmente por Gina Carrioni (1991), es importante para las comunidades establecidas en La Chorrera, pero es contradictorio con estas mismas comunidades. Después de cerca de 80 años de una educación impuesta, donde todo lo peor era del indígena:

\footnotetext{
${ }^{1}$ Este escrito es producto de conversaciones entre los dos autores en el contexto de la Maloka.

2 Coordinador de Comisión de Ordenamiento de AZICATCH (Asociación Zonal Indígena de Cabildos y Autoridades Tradicionales de La Chorrera, Amazonas)

${ }^{3}$ Coordinador Proyección Social UPN CERES La Chorrera
} 
su idioma, pensamiento, vestido, gestos; se consideraba inculto, negativo, afín a la idolatría; aparece entonces el tema de que lo propio es lo que hay que valorar, lo que choca con la opinión de muchos padres de familia en la región, muy pocos aceptaron esa reivindicación y por eso los esfuerzos hechos por algunos docentes siempre encontraron resistencia.

Por eso el avance que se pensó no dio los resultados que esperábamos tener en el momento; entre las razones encontramos: se le entregó una responsabilidad al docente indígena de la cual no tenía suficiente conocimiento, porque tenía una formación académica de occidente, entonces algunos inventaron, improvisaron y en el peor de los casos, mintieron. Porque no existía un concepto definido de qué era etnoeducación, no se sabía la diferencia entre propio y ajeno: simplemente la enseñanza académica se daba en idioma vernáculo. Esto generó mucha resistencia entre los mayores, los ancianos y buen número de jóvenes, que no lo vieron bien, específicamente con los estudiantes que salieron a estudiar y regresaron. A esto se agrega el efecto desfavorable que representaba para los hablantes de lenguas minoritarias el hecho de ser ridiculizados por los hablantes de la lengua mayoritaria.

Hay una propuesta ambiciosa donde se mira que el problema es formar docentes indígenas en etnoeducación y se logra establecer un nivel aceptable a nivel de básica primaria, con todas las dificultades que podamos imaginarnos: falta de materiales didácticos, infrastructura, presupuesto. La propuesta tenía el fin de mejorar las condiciones que ofreció el decreto 1142, que no eran las mejores (el hecho de ser indígena no quiere decir que sea buen docente). Ante la imposibilidad de continuar con la actualización de docentes indígenas, se opta por un error grandísimo: continuar con los programas estatales (escuela nueva, promoción automática), lo que genera una confusión entre docentes indígenas, con un futuro incierto ¿qué es lo que estamos aplicando?

En la secundaria el panorama es semejante: aunque existe un PEI muy elaborado, después de 10 años no se ha hecho ninguna revisión; los docentes indígenas con título de licenciados en etnoeducación parten de una concepción teórica que está lejos de la aplicación pedagógica propia. Como prueba de ello, no existe el intento de tener materiales didácticos propios para su aplicación. Otra realidad notable es que pocos docentes aplican lo establecido en el PEI, haciendo que los estudiantes no sean competitivos, lo que se demuestra en las pruebas de estado y su comportamiento dentro y fuera de la institución, existiendo excepciones entre profesores y alumnos.

Ante esta realidad, es obligatorio actualizar, capacitar a nuestros docentes con temas de pedagogía y didáctica, aprovechando la presencia de una Universidad Pedagógica en el medio, incentivándolos en especializaciones, maestrías con una real etnoeducación que parta de la región, de su tradición, con sus mayores como orientadores, quienes guían desde sus espacios tradicionales, los mambiaderos, las generaciones que están encargadas de continuar con el cuidado de la Amazonía y sus culturas

Para los mayores, la etnoeducación no es una preocupación, la preocupación es que tantos métodos de enseñanza que se han ofrecido han conducido al desorden. La educación propia que imparten los mayores desde el mambiadero sigue su método de enseñanza sin los vaivenes de las modas pedagógicas. Una investigación sobre los dispositivos de 
socialización secundaria (Rodríguez, 2001) profundiza la parte conceptual de etnodidáctica en la zona. La definición de Artunduaga (1997) es: la etnoeducación, como sistema, constituye un proceso a través del cual los miembros de un pueblo internalizan y construyen conocimientos y valores, y desarrollan habilidades y destrezas de acuerdo con sus características, necesidades, aspiraciones e intereses culturales, que les permiten desempeñarse adecuadamente en su medio y proyectarse con identidad hacia otros grupos humanos.

Una concertación del currículo con los ancianos, jóvenes y otros miembros de la comunidad para la actual Licenciatura que se ofrece en La Chorrera, contempla en su proyecto en todos los ambientes de formación espacios que apoyan directamente este proceso, como: Cosmovisión, Naturaleza y plan de vida; Tecnologías de la información y de la Comunicación; Territorio.

Cultura e identidad; Diversidad y Organización Biológica. Los rituales han mostrado ser un gran sustento para la educación en la cultura; la presencia de la academia en estos espacios de intercambio, como los bailes tradicionales, es etnoeducación desde lo intercultural.

Pensar - palabra - obra. En la formación de la educación tradicional de la cultura del tabaco, están reguladas cuatro etapas del conocimiento y de la vida: escuchar, practicar, enseñar y cuidar.

Escuchar. Todo padre o madre tiene una educación para recibir la futura generación de su pueblo y de su clan, para que el futuro heredero escuche el conocimiento de dos maneras, indirecta, en tiempo de gestación y es responsabilidad de la madre; directa, escucha el concejo en su niñez por parte de su familia, abuelos, tíos, padres.

Practicar. Durante la juventud, el joven practica lo escuchado desde niño, haciendo y diciendo siempre la verdad.

Enseñar. Es deber de todo padre o madre enseñar el consejo a sus hijos, nietos y bisnietos en la protección y el cuidado de la vida de él y sus entornos. A decir lo más sencillo que es 'papá', 'mamá', 'abuela', 'abuelo'. Así no sea su propio tío, se le debe decir así a cualquier mayor que no sea su hermano o su padre.

Cuidar. Todo abuelo debe proteger su enseñanza ,que es el consejo para que su generación sea trabajadora, honrada, obediente y respetuosa. Solo así se inmuniza de las enfermedades materiales y espirituales, que es el deseo de todo abuelo, que su generación se multiplique bajo la protección del tabaco, la coca y la yuca dulce., es decir, nuestra cultura nunca termina de formar, trabajar, enseñar; nunca se sabe todo, nadie lo sabe todo, excepto Moo, dueño del conocimiento universal. Dietada esta palabra es lo que le da autoridad y poder; la autoridad no es una persona, es la palabra, es un canasto.

\section{El pensamiento}

Nana Ta+no jag+y+ mona komuide: todo lo que existe surgió de lo intangible; ésta es la base del pensamiento propio de los hijos del tabaco, la coca y la yuca dulce. En su proceso de perfección o evolución se vuelve real, tangible. Esto está reflejado en relatos, mitos, leyendas, rituales, creencias, establecido en el uso y manejo del espacio y el tiempo, celosamente protegido por todos los ancianos (eikome) de cada clan o de cada pueblo, de 
manera oral y milenaria. La expresión de este concepto se observa en el calendario étnico, que condensa todo el conocimiento de la cultura debidamente ordenado de manera natural, que se vuelve en el poder de la palabra que enseñan los eikome cada noche en el mambiadero. La función de los ancianos es, entonces, mantener vivo el pensamiento de la cultura y darle responsabilidad a la palabra, que es lo que da autoridad a las personas que cumplen la dieta del consejo (escuchar, practicar, enseñar y cuidar).

El término jag+y+ para la cultura está realmente en el corazón, como sentimiento, pero se denota como 'pensamiento' para dialogar con una cultura que es hija de la razón. El sentimiento es la madurez del pensamiento. Para el anciano no existe dualidad: todo lo recibe bien, así esté mal; cuando recibe un enfermo, dice que está bien, porque él lo va a sanar.

Este es el deber de un buen anciano: cuidar esta parte de la vida indígena.

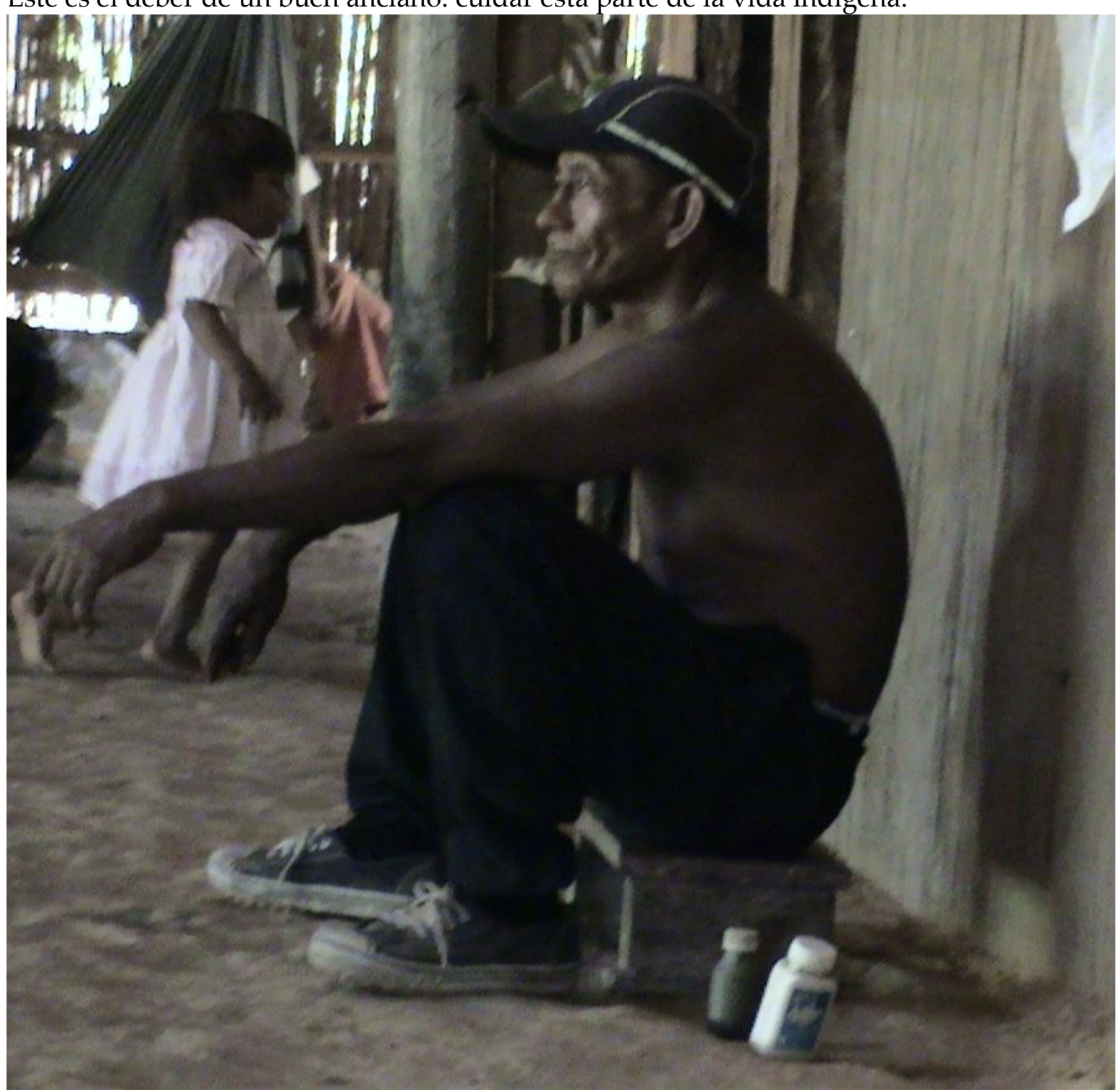

\section{CALENDARIO ÉTNICO}

Este no es producto del sentimiento, sino del pensamiento, porque el ta+no jag+y+ es Moo, dios creador, por esto la expresión Nana Ta+no jag+y+ mona komuide. Lo cierto es que el 
calendario étnico es obra de Moo entregado a Kome, el humano, con todo el ordenamiento establecido por Moo, no por humano, por esto no falla, no tiene error. En etnoeducación, el calendario es eño (maloca o madre) universal, protectora del tiempo y el espacio, que interpretado en una sola expresión sería el 'aire de vida', que tiene incorporado en su esencia el trabajo, la abundancia y la humanización del pensamiento indígena, que debemos enseñar a vivir en el diario convivir entre lo divino, lo humano y la naturaleza (Moo, kome y jinobik+).

A partir de esta concepción, el calendario étnico de la cultura del tabaco expresa la integralidad de las actividades humanas con la naturaleza y sus elementos (agua, tierra, peces y seres del agua, animales terrestres, aves, bosque) y los fundamentos y principios espirituales que orientan el manejo y relación con este mundo en relación al tiempo y el espacio, concebido desde las culturas uitoto, bora, okaina y muinane. Esta relación se hace explícita en el Plan de Vida (Azicatch 2007).

Una visión intangible y espiritual se vuelve tangible en el calendario tradicional:

\section{CALENDARIO DE MANEJO AMBIENTAL DE LOS HIJOS DEL TABACO, COCA Y YUCA DULCE}

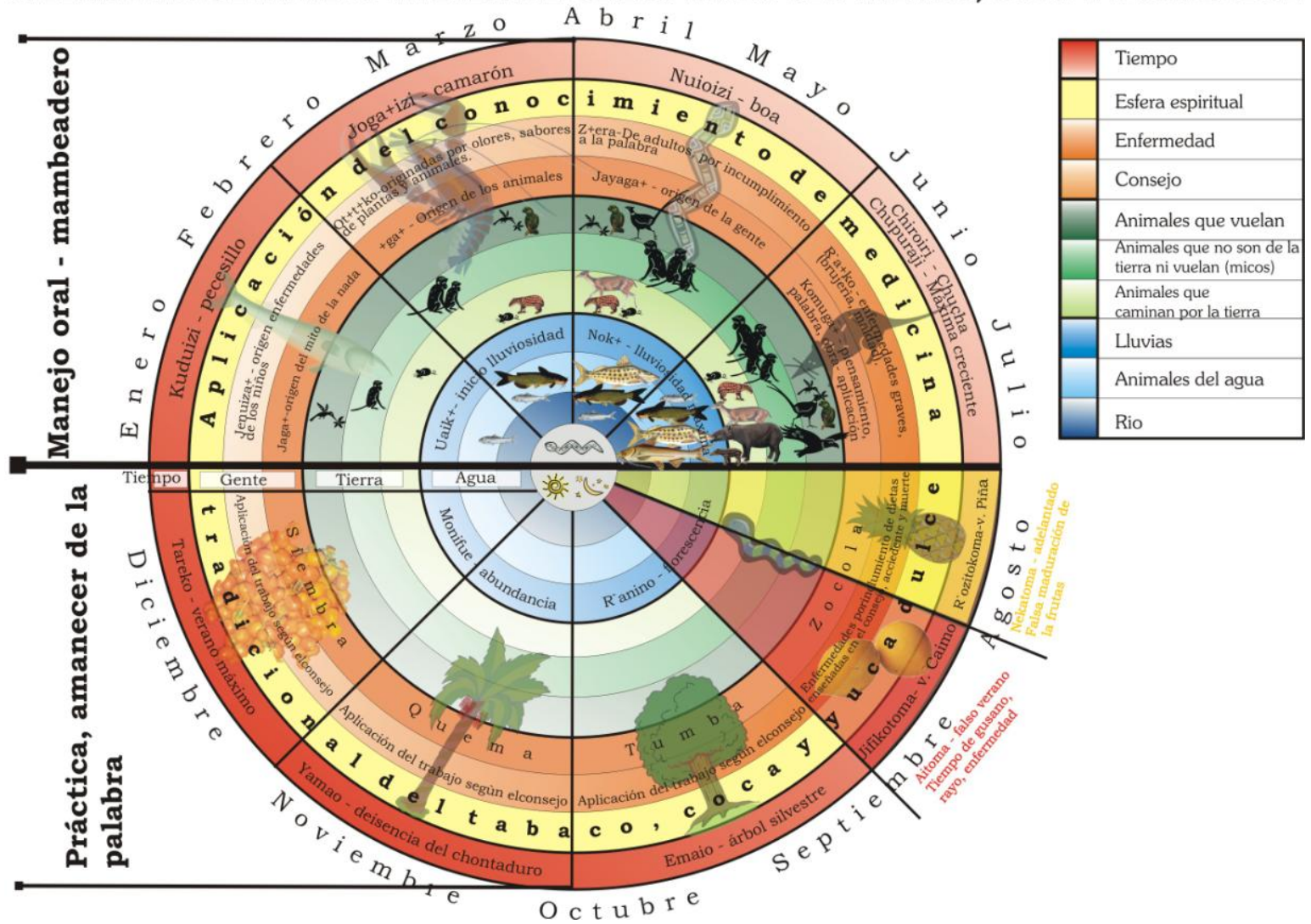

El calendario está hecho en anillos concéntricos porque es un sistema que cierra un círculo. Cada cierre del círculo es un cierre de ciclo, que inicia en el rozi (friaje, las heladas). Cada ciclo enseña el comportamiento con relación al comportamiento humano, que debe enseñarse diariamente, para no cometer errores con eño. Sabemos, entonces, que no puede haber verano en mayo, que es tiempo de rozi y por lo tanto no podríamos hacer actividades de pesca con barbasco, porque por más que apliquemos barbasco, no sería suficiente por la cantidad de agua y lluvia en este tiempo; si no recibimos esta enseñanza, nos moriríamos de 
hambre sin un pez para llevar a nuestro plato. En esta época utilizaríamos el tapaje, que consiste en cercar con hojas de palma los rebalses o zonas inundadas para atrapar gran cantidad de peces. Cada ciclo establece una preparación y una práctica consecuente en el siguiente orden: la mitad del tiempo del año es seco $f+m o n a$, medido por los veranos de piña, caimo, guamo, emaio, chontaduro (rozikotoma, jifikotoma, jizaio, emaio, yamao -cuando el verano se alarga, se dice que hay tareko-). Al final del rozi hay un verano falso aitoma, porque calienta y llueve; este periodo se caracteriza por repentina lluvia, truenos, viento, lo que significa la multiplicación de la mayoría de los gusanos malos, lo que altera el organismo humano, produciendo enfermedades; la hojarasca del río produce olores ofensivos a los niños; los zancudos pululan; es común la malaria y el vómito; la mayoría de los ancianos mueren en este tiempo. El tratamiento es preventivo; el calendario previene: para la calentura se cura yerbafría, para brotes achiote, para los dolores ortiga; se cura juñoi (manicuera, jugo de yuca dulce) para dar fortaleza en tiempos difíciles. Los niños y mujeres deben llegar más temprano a casa en la tarde. Se debe bañar antes de que canten los pájaros, evitando así el agua contaminada y dando fortaleza del espíritu; Moo no permanece en lo que está contaminado. Esta enseñanza va para toda la vida.

El tiempo húmedo en el calendario está medido por los friajes. Comienza con el friaje de sambico kuduizi que coincide con enero-febrero, continúa con el friaje de joga+izi camarón, el de boa nuioizi termina con el de chucha chiroiri. En cada friaje se intensifica más las lluvias y por ende el nivel de los cuerpos agua hasta llegar al mes de julio, cuando empieza a descender la intensidad de lluvias en el tiempo de $f+m o n a$.

Tabla 1. Explicación de los niveles del calendario étnico de los hijos del tabaco.

\begin{tabular}{|c|c|c|c|c|}
\hline Niveles & Plan de Vida & $\begin{array}{ll}\text { Plan de } \\
\text { Abundancia }\end{array}$ & Interpretación & \\
\hline Origen & \multirow[t]{2}{*}{$\begin{array}{l}\text { Uai - palabra de } \\
\text { vida }\end{array}$} & \multirow[t]{2}{*}{$\begin{array}{l}\text { ambiental } \quad y \\
\text { espiritualidad }\end{array}$} & $\begin{array}{l}\text { la oscuridad } \\
\text { trasciende a la } \\
\text { luz }\end{array}$ & \\
\hline Tiempo & & & $\begin{array}{l}\text { ciclos del sol y la } \\
\text { luna, verano y } \\
\text { friaje }\end{array}$ & \\
\hline Agua -peces & Menig+ buinaima & $\begin{array}{l}\text { manejo integral } \\
\text { del agua - } \\
\text { acuicultura }\end{array}$ & \multirow{3}{*}{$\begin{array}{l}\text { etapas } \\
\text { evolución de } \\
\text { sueño } \\
\text { creador en } \\
\text { el espíritu toma } \\
\text { forma humana }\end{array}$} & \multirow{3}{*}{$\begin{array}{l}\infty \\
0 \\
0 \\
0 \\
\pi \\
\infty \\
0 \\
0 \\
\pi \\
\pi\end{array}$} \\
\hline Bosque & Yua buinaima & $\begin{array}{l}\text { chagra } \\
\text { agricultura - } \\
\text { manejo de flora - } \\
\text { canciones de } \\
\text { frutas }\end{array}$ & & \\
\hline $\begin{array}{l}\text { Animales } \\
\text { Aves- } \\
\text { trepadores } \\
\text { (micos) }\end{array}$ & $Z+k+d a$ buinaima & manejo fauna & & \\
\hline Hombre & D+ona & lo que enseña y & materialización & \\
\hline
\end{tabular}




\begin{tabular}{|c|c|c|c|}
\hline & normas de vida & $\begin{array}{l}\text { practica } \\
\text { (etnoeducación) }\end{array}$ & $\begin{array}{l}\text { de lo intangible } \\
\text { (Moo a kome), }\end{array}$ \\
\hline Conocimiento & Yetarafue consejo & $\begin{array}{ll}\text { trabajo } & - \\
\text { obediencia } & - \\
\text { honestidad } & - \\
\text { respeto } & \end{array}$ & $\begin{array}{lr}\text { enseñar } & \text { el } \\
\text { manejo } & \text { y } \\
\text { orientar } & \text { la } \\
\text { práctica } & - \\
\text { etnoeducación } \\
\text { gobierno de } \\
\begin{array}{ll}\text { servicio, no de } \\
\text { opresión }\end{array} \\
\end{array}$ \\
\hline Enfermedades & $\begin{array}{l}\text { Incumplimiento } \\
\text { de normas de } \\
\text { vida }\end{array}$ & $\begin{array}{ll}\text { oraciones } & y \\
\text { conjuros } & \end{array}$ & $\begin{array}{l}\text { justicia, premio } \\
\text { o castigo }\end{array}$ \\
\hline Espiritual & $\begin{array}{l}\text { Diferencia del } \\
\text { comportamiento } \\
\text { animal y humano }\end{array}$ & $\begin{array}{l}\text { rituales - bailes - } \\
\text { cantos sagrados } \\
\text { (no de baile) }\end{array}$ & $\begin{array}{l}\text { tabaco }(\text { Moo }) \text {, } \\
\text { coca (komo) y } \\
\text { yuca dulce }(e \tilde{n} o)\end{array}$ \\
\hline
\end{tabular}

\section{La palabra}

El poder de la palabra de la autoridad de un clan (en algunos casos especiales de algunos clanes en conjunto), tiene poder porque se dieta. La Dieta consiste en no cometer lo prohibido por la palabra de la coca, el tabaco y la yuca dulce, además de escuchar, practicar, enseñar, cuidar el concejo; además, no incurrir en $i k+$ rafue, j+rufue, yog+rafue, urafue, yaguerofe (ira, lujuria, chisme, indirectas, burla), quitarle al huérfano lo que le corresponde, mentir, vicios. El poder de la palabra exige también ser trabajador, cumplir con la palabra (promesas) y ser obediente, sólo así se es respetuoso consigo mismo y con los demás, entonces así su clan le obedece, será un buen gobernante. La palabra rige para todos los niveles: docente (numairama), cazador, cantor, niño, curandero, orientador, joven, mujer, todos.

\section{La obra}

Consiste en manejar cada uno de los niveles y espacios en el tiempo correspondiente del calendario étnico (teoría) y ecológico (aplicación). La obra está en la aplicación de la enseñanza de la palabra. La obra es la suma del pensamiento más la palabra que debe ser coherente todo el tiempo porque no se puede enseñar de chagra sin tener chagra, no debes hablar de tabaco, coca y yuca dulce cuando no lo tiene en propiedad, no puedes decir que no robes cuando robas, porque así queda viciada la palabra del tabaco y queda sin autoridad, así tenga las toneladas de coca y de ambil.

\section{BIBLIOGRAFÍA}

- Artunduaga, L. A. (1997). La etnoeducación: una dimensión de trabajo para la educación en comunidades indígenas de Colombia. Revista Iberoamericana de Educación Número 13 Educación Bilingüe Intercultural 
- Azicatch. (2007). El abuelo tabaco. Plan de vida y el ordenamiento de los hijos del tabaco, la coca la yuca dulce. ms.

- Carrioni, G. (1991). Significado de la Etnoeducación, sus limitaciones y alcances en Colombia. En Serie Proyectos $N^{\circ} 6$ Bogotá, CINDE.

- Rodríguez-R, Á. (2001). Dispositivos de Socialización Secundaria en comunidades indígenas Huitoto. Bases para una etnodidáctica. Resumen de la investigación. Caciques indígenas, COLCIENCIAS, FUCAI. 